\title{
Next-to-leading-logarithm threshold resummation for exclusive $B$ meson decays
}

\author{
Zhi-Qing Zhang ${ }^{1, \mathrm{a}}$, Hsiang-nan $\mathbf{L i}^{2, \mathrm{~b}}$ \\ ${ }^{1}$ Department of Physics, Henan University of Technology, Zhengzhou 450052, Henan, People's Republic of China \\ ${ }^{2}$ Institute of Physics, Academia Sinica, Taipei, Taiwan 115, Republic of China
}

Received: 10 September 2020 / Accepted: 24 June 2021 / Published online: 8 July 2021

(c) The Author(s) 2021

\begin{abstract}
We extend the threshold resummation of the large logarithms $\ln x$ which appear in factorization formulas for exclusive $B$ meson decays, $x$ being a spectator momentum fraction, to the next-to-leading-logarithm (NLL) accuracy. It is shown that the NLL resummation effect provides suppression in the end-point region with $x \sim 0$ stronger than the leading-logarithm (LL) one, and thus improves perturbative analyses of the above processes. We revisit the $B \rightarrow K \pi$ decays under the NLL resummation in the perturbative QCD approach, and find that it induces up to $20 \%$ variation of the direct $\mathrm{CP}$ asymmetries in various modes relative to the LL results. Our method to avoid the Landau singularity in the inverse Mellin transformation causes at most $10 \%$ theoretical uncertainty.
\end{abstract}

\section{Introduction}

Factorization theorems have been one of the major theoretical approaches to exclusive $B$ meson decays, in which a decay process is factorized into a convolution of a hard kernel with hadron distribution amplitudes. A crucial issue on the application of factorization theorems to a key ingredient of these decays, $B$ meson transition form factors, is the endpoint singularity, which appears as a spectator parton carries a vanishing momentum fraction $x \rightarrow 0$ [1-3]. Because of this end-point singularity, a $B$ meson transition form factor is treated as a nonperturbative input in the QCD-improved factorization approach [4-6] based on the collinear factorization theorem. In the soft-collinear effective theory the end-point singularity can be removed by the zero-bin subtraction [7], so that a $B$ meson transition form factor becomes factorizable in the collinear factorization. It was argued that a parton transverse momentum $k_{T}$ is not negligible, when the end-

\footnotetext{
a e-mail: zhangzhiqing@haut.edu.cn (corresponding author)

b e-mail: hnli@phys.sinica.edu.tw
}

point region is important. The perturbative QCD (PQCD) approach based on the $k_{T}$ factorization theorem was then proposed [8-13], in which the end-point singularity is regularized by a parton $k_{T}$, and a $B$ meson transition form factor also becomes factorizable [14].

An alternative removal of the end-point singularity in the framework of the collinear factorization has been suggested in [15]. When the end-point region dominates, the double logarithms $\alpha_{s} \ln ^{2} x$ from radiative corrections [2,16], $\alpha_{s}$ being the strong coupling constant, should be organized to all orders to improve perturbative expansion. The first systematic study was done in [15], where these double logarithms were factorized from exclusive $B$ meson decays into a universal jet function, and resummed up to the leading-logarithm (LL) accuracy. It was then shown that the resultant jet function vanishes quickly at $x \rightarrow 0$, and suppresses the end-point singularities in the $B \rightarrow \pi$ form factors. The threshold resummation effect on more complicated two-body hadronic $B$ meson decays, which involve the annihilation and nonfactorizable amplitudes in addition to the factorizable ones proportional to transition form factors, was analysed in [17] and implemented in the PQCD approach widely afterwards.

In this paper we will extend the LL threshold resummation performed in [15] to the next-to-leading-logarithm (NLL) accuracy. To accomplish this task, we calculate the jet function stated above at one loop to identify the complete large logarithms, solve an evolution equation for the jet function in the Mellin space to get all-order summation of the logarithms, match the all-order summation to the one-loop result to determine the initial condition of the jet function, and follow the best fit method in [14] to obtain the threshold resummation in the momentum fraction $x$ space. It will be demonstrated that the NLL jet function exhibits suppression at the end point $x \sim 0$ stronger than the LL one. Because the threshold resummation modifies hard decay kernels by including partial higher order contributions, hadron distribution amplitudes, such as the uncertain $B$ meson distribution 
amplitude, should be adjusted accordingly to maintain the values of $B$ meson transition form factors [18]. Therefore, we compare the LL and NLL resummation effects by investigating their impacts on the $\mathrm{CP}$ asymmetries in the $B \rightarrow K \pi$ decays, which are less sensitive to choices of hadron distribution amplitudes. It is found that the replacement of the LL jet function by the NLL one in the PQCD factorization formulas causes up to $20 \%$ variation of the direct $\mathrm{CP}$ asymmetries in various $B \rightarrow K \pi$ modes, an effect which needs to be taken into account for precision calculations of $B$ meson decays.

In Sect. 2 we compute the one-loop jet function, derive its evolution equation and the corresponding evolution kernels in the Mellin space, and solve the evolution equation to attain the NLL threshold resummation. The best fit method is employed to transform the jet function from the Mellin space back to the momentum fraction space in Sect. 3. It is verified that the Landau singularity in the inverse Mellin transformation can be avoided, and the theoretical uncertainty is at most $10 \%$ and under control in the above method. The impacts of the LL and NLL resummations on the CP asymmetries in the $B \rightarrow K \pi$ decays are then examined and compared. Section 4 contains the conclusion and outlook.

\section{Threshold resummation}

\subsection{One-loop quark diagrams}

The definition of the jet function $J(x)$ in terms of a quark field and its associated Wilson link, which is constructed from the factorization of the radiative decay $B\left(P_{1}\right) \rightarrow \gamma\left(P_{2}\right) l \bar{\nu}$, is referred to [15]. The Wilson link runs in the direction $n$, that contains the arbitrary components $n^{+}$and $n^{-}$. The quark momentum has been parametrized as $P_{2}-k$, where the photon momentum $P_{2}$ is in the minus direction and the momentum $k=\left(x P_{1}^{+}, 0, \mathbf{0}_{T}\right)$ of the light quark in the $B$ meson is in the plus direction. That is, this quark is slightly off-shell by $\left(P_{2}-k\right)^{2}=-2 x P_{1}^{+} P_{2}^{-} \equiv-x Q^{2}$. The leading-order (LO) jet function has been chosen as $J^{(0)}(x)=1$. The one-loop vertex correction in Fig. 1 is written as

$$
\begin{aligned}
J_{a}^{(1)}(x)= & -i g^{2} C_{F} \mu^{2 \epsilon} \int \frac{d^{4-2 \epsilon} l}{(2 \pi)^{4-2 \epsilon}} t r \\
& {\left[\frac{p_{+} \nu_{-}}{4} \gamma_{\beta} \frac{P_{2}-k+l}{\left(P_{2}-k+l\right)^{2}}\right] \frac{n^{\beta}}{n \cdot l l^{2}}, }
\end{aligned}
$$

where $C_{F}=4 / 3$ is a color factor, $\mu$ is the renormalization scale, and $l$ is the gluon momentum. The projector $p_{+} p_{-} / 4$, with the light-like vectors $v_{+}=\left(1,0, \mathbf{0}_{T}\right)$ and $v_{-}=\left(0,1, \mathbf{0}_{T}\right)$, arises from the factorization of the jet function [15]. A straightforward evaluation gives

$J_{a}^{(1)}(x)=-\frac{\alpha_{S} C_{F}}{4 \pi}\left(-\frac{1}{\epsilon}-\ln \frac{4 \pi \mu^{2}}{x Q^{2}}+\ln ^{2} \frac{Q^{2} x}{\xi^{2}}\right.$
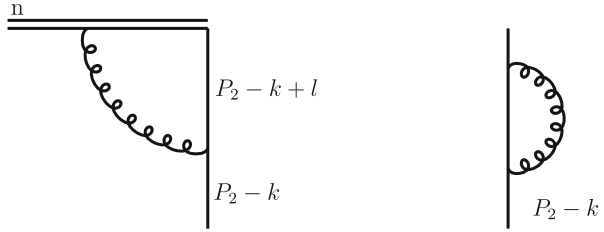

Fig. 1 One-loop diagrams for the jet function, where the double line represents the Wilson link

$$
\left.+\ln \frac{Q^{2} x}{\xi^{2}}+\frac{4}{3} \pi^{2}+\gamma_{E}-2\right)
$$

for $n^{2}>0$, with the $n$-dependent factor $\xi^{2} \equiv 4\left(P_{2} \cdot n\right)^{2} / n^{2}$ and the Euler constant $\gamma_{E}$. Note that the jet function depends on the Lorentz invariants $\left(P_{2}-k\right) \cdot n \approx P_{2} \cdot n$ in the small $x$ limit and $n^{2}$, and that the Feynman rules associated with the Wilson link shows a scale invariance in $n$. These facts explain why the two vectors $P_{2}-k$ and $n$ appear via the ratio $\xi^{2}$ in Eq. (2).

The self-energy correction in Fig. 1 is expressed as

$$
\begin{aligned}
J_{b}^{(1)}(x)= & -i g^{2} C_{F} \mu^{2 \epsilon} \int \frac{d^{4-2 \epsilon} l}{(2 \pi)^{4-2 \epsilon}} t r \\
& \times\left[\frac{p_{+} \nu_{-}}{4} \gamma^{\nu} \frac{P_{2}-k-l}{\left(P_{2}-k-l\right)^{2}} \gamma_{\nu} \frac{P_{2}-k}{\left(P_{2}-k\right)^{2}}\right] \frac{1}{l^{2}} \\
= & -\frac{\alpha_{s} C_{F}}{4 \pi}\left(\frac{1}{\epsilon}+\ln \frac{4 \pi \mu^{2}}{x Q^{2}}-\gamma_{E}+2\right) .
\end{aligned}
$$

We then have the $\mathcal{O}\left(\alpha_{s}\right)$ jet function

$$
\begin{aligned}
J^{(1)}(x)= & J_{a}^{(1)}(x)+J_{b}^{(1)}(x)=-\frac{\alpha_{s} C_{F}}{4 \pi} \\
& \times\left(\ln ^{2} \frac{Q^{2} x}{\xi^{2}}+\ln \frac{Q^{2} x}{\xi^{2}}+\frac{4}{3} \pi^{2}\right),
\end{aligned}
$$

which is independent of $\mu$, i.e., ultraviolet finite.

We apply the Mellin transformation from the momentum fraction $x$ space to the moment $N$ space

$\tilde{J}(N) \equiv \int_{0}^{1} d x(1-x)^{N-1} J(x)$

It implies that the transformed jet function $\tilde{J}(N)$ at large $N$ collects the contribution mainly from the small $x$ region. The Mellin transformation of the LO jet function, $\tilde{J}^{(0)}(N)=$ $1 / N$, is trivial. The Mellin transformation of Eq. (4) yields, in terms of the variable $\bar{N} \equiv N \exp \left(\gamma_{E}\right)$,

$\tilde{J}^{(1)}(N) \approx-\frac{\alpha_{s} C_{F}}{4 \pi}\left(\ln ^{2} \frac{Q^{2}}{\xi^{2} \bar{N}}+\ln \frac{Q^{2}}{\xi^{2} \bar{N}}+\frac{3}{2} \pi^{2}\right) \frac{1}{N}$,

in the large $N$ limit up to corrections down by powers of $1 / N$. 


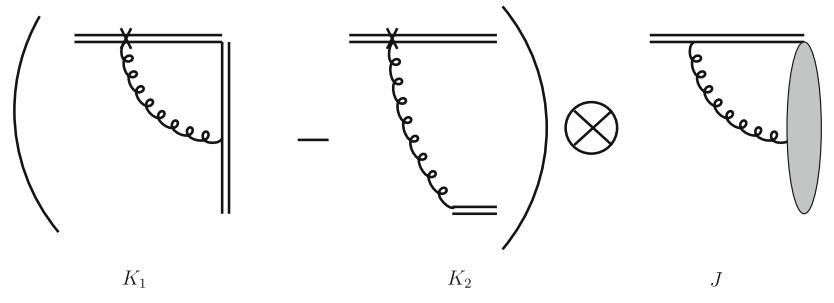

Fig. 2 Convolution of the $\mathcal{O}\left(\alpha_{s}\right)$ soft function $K$ with the jet function $J$, where the symbol $\times$ represents the special vertex

\subsection{Evolution equation for $J$}

As indicated by the above one-loop calculation, the important logarithms in the jet function depend on the factor $\xi^{2} \equiv 4 v^{2} P_{2}^{-2}$. To resum these logarithms, we construct the evolution equation for the jet function $[19,20]$

$2 v^{2} \frac{d J}{d v^{2}}=-\frac{n^{2}}{v_{-} \cdot n} v_{-\alpha} \frac{d J}{d n^{\alpha}}$.

The derivative respect to $n^{\alpha}$ applies to the Feynman rules of the Wilson link, generating

$$
-\frac{n^{2}}{v_{-} \cdot n} v_{-\alpha} \frac{d}{d n^{\alpha}} \frac{n^{\beta}}{n \cdot l}=\frac{\hat{n}^{\beta}}{n \cdot l},
$$

with the special vertex

$\hat{n}^{\beta}=\frac{n^{2}}{v_{-} \cdot n}\left(\frac{v_{-} \cdot l}{n \cdot l} n^{\beta}-v_{-}^{\beta}\right)$.

The technique of varying Wilson links has been applied to the resummation of various types of logarithms, such as the rapidity logarithms in the $B$ meson wave function [21], and the joint logarithms in the pion wave function [22]. The Ward identity for the summation over the special vertices leads to the factorization of the soft function $K$ and the hard function $G$ from the derivative of the jet function $[19,20]$

$2 \nu^{2} \frac{d J}{d \nu^{2}}=(K+G) \otimes J$.

Figure 2 depicts the factorization of the soft function $K$ at $\mathcal{O}\left(\alpha_{s}\right)$, which contains two pieces $K_{1}$ and $K_{2}$. The former is written as

$K_{1}=-i g^{2} C_{F} \mu^{2 \epsilon} \int \frac{d^{4-2 \epsilon}}{(2 \pi)^{4-2 \epsilon}} \frac{\hat{n}_{\mu}}{n \cdot l} \frac{g^{\mu \nu}}{l^{2}-m^{2}} \frac{P_{J v}}{P_{J} \cdot l}-\delta K$,

with the momentum $P_{J}=P_{2}-k$, where the gluon mass $m^{2}$, serving as an infrared regulator, will approach to zero eventually. Choosing the additive counterterm

$\delta K=-\frac{\alpha_{s} C_{F}}{2 \pi}\left[\ln \left(4 \pi v^{2}\right)+\frac{1}{\epsilon}-\gamma_{E}\right]$, we have

$K_{1}=-\frac{\alpha_{s} C_{F}}{2 \pi} \ln \frac{\mu^{2}}{v^{2} m^{2}}$.

The loop momentum $l$ flows through the jet function for $K_{2}$, so they appear in a convolution

$K_{2} \otimes J=i g^{2} C_{F} \int \frac{d^{4} l}{(2 \pi)^{4}} \frac{\hat{n}_{\mu}}{n \cdot l} \frac{g^{\mu \nu}}{l^{2}-m^{2}} \frac{P_{J v}}{P_{J} \cdot l} J\left(x-\frac{l^{+}}{P_{1}^{+}}\right)$.

Performing the contour integration over $l^{-}$for $-(1-x) P_{1}^{+}<$ $l^{+}<0$ followed by the integration over the transverse momentum $l_{T}$, and employing the variable change $u=$ $x-l^{+} / P_{1}^{+}$, we arrive at

$$
\begin{aligned}
K_{2} \otimes J= & \frac{\alpha_{s} C_{F}}{\pi}\left[\int_{x}^{1} d u \frac{J(u)-J(x)}{u-x}\right. \\
& \left.+\int_{x}^{1} d u \frac{(u-x) J(x)}{(u-x)^{2}+M^{2}}\right],
\end{aligned}
$$

with the infrared regulator $M^{2} \equiv v^{2} m^{2} / P_{1}^{+2}$.

The Mellin transformation of the first integral in Eq. (15) gives

$$
\begin{aligned}
& \int_{0}^{1} d x(1-x)^{N-1} \int_{x}^{1} d u \frac{J(u)-J(x)}{u-x} \\
& \approx \int_{0}^{1} d u J(u) \int_{0}^{1} d t \frac{(1-t)^{N-1}}{t}\left(\frac{1}{1-t}-u\right)^{N-1} \\
& \quad-\int_{0}^{1} d x(1-x)^{N-1} J(x) \int_{0}^{1} \frac{d t}{t}
\end{aligned}
$$

where the order of the integrations over $x$ and $u$ has been exchanged in the first term, the variable change $x=u(1-t)$ has been applied, and the upper bound $1-x$ of the integration variable $t$ in the second term has been approximated by 1 . This approximation holds up to an infrared finite constant, which will be compensated by matching later. The further approximation $[1 /(1-t)-u]^{N-1} \approx(1-u)^{N-1}$, which holds in the dominant small $t$ region, brings Eq. (16) into

$\tilde{J}(N) \int_{0}^{1} d t \frac{(1-t)^{N-1}-1}{t} \approx-\tilde{J}(N) \ln \bar{N}$.

The above result is subject to corrections down by a power of $1 / N$.

We also apply the Mellin transformation to the second integral in Eq. (15):

$$
\begin{aligned}
& \int_{0}^{1} d x(1-x)^{N-1} J(x) \int_{0}^{1} d u \frac{(u-x)}{(u-x)^{2}+M^{2}} \\
& \quad \approx \tilde{J}(N) \ln \frac{1}{M} .
\end{aligned}
$$

The sum of Eqs. (17) and (18) yields the Mellin transformation of Eq. (15), 

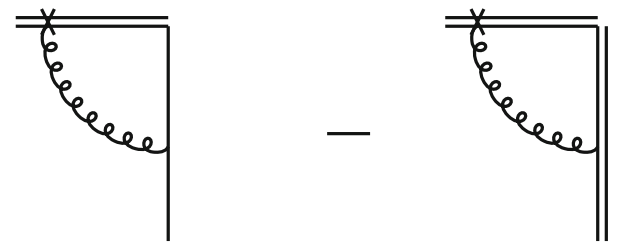

Fig. $3 \mathcal{O}\left(\alpha_{s}\right)$ hard function $G$

$$
\begin{aligned}
& \int_{0}^{1} d x(1-x)^{N-1} K_{2} \otimes J \\
& =-\frac{\alpha_{s} C_{F}}{\pi}\left(\ln \bar{N}-\ln \frac{P_{1}^{+}}{\nu m}\right) \tilde{J}(N),
\end{aligned}
$$

which is then combined with Eq. (13) into

$\int_{0}^{1} d x(1-x)^{N-1} K \otimes J=-\frac{\alpha_{s} C_{F}}{\pi} \ln \frac{\mu \bar{N}}{P_{1}^{+}} \tilde{J}(N)$.

It is seen that the infrared regulator $m^{2}$ has disappeared in the above combination.

The first diagram for the $\mathcal{O}\left(\alpha_{s}\right)$ hard function $G$ in Fig. 3 contributes

$$
\begin{aligned}
G_{1}= & -i g^{2} C_{F} \int \frac{d^{4} l}{(2 \pi)^{4}} \operatorname{tr}\left[\frac{p_{+} \nu_{-}}{4} \gamma_{v} \frac{P_{2}-k+/ l}{\left(P_{2}-k+l\right)^{2}}\right] \\
& \times \frac{g^{\mu \nu}}{l^{2}-m^{2}} \frac{\hat{n}_{\mu}}{n \cdot l}=-\frac{\alpha_{s} C_{F}}{2 \pi} \ln \frac{4 v^{2} P_{2}^{-2}}{m^{2} e} .
\end{aligned}
$$

We have dropped the small momentum $k$, to which the hard function is not sensitive. Instead, the infrared regulator $m^{2}$ is introduced, whose dependence will be removed by the subtraction below. Note that the above expression is free of an ultraviolet divergence. The final result for $G$ in Fig. 3 is

$G=G_{1}-K_{1}-\delta G=-\frac{\alpha_{s} C_{F}}{\pi} \ln \frac{2 \nu^{2} P_{2}^{-}}{\sqrt{e} \mu}$,

with the additive counterterm $\delta G=-\delta K$, where the subtraction $K_{1}$ avoids double counting of the soft contribution.

\subsection{Solution to evolution equation}

The functions $K$ and $G$ involve only single soft and ultraviolet logarithms, respectively, so they can be treated by renormalization-group $(\mathrm{RG})$ methods:

$\mu \frac{d}{d \mu} K=-\lambda_{K}=-\mu \frac{d}{d \mu} G$,

where the anomalous dimension $\lambda_{K}=\mu d \delta K / d \mu$ is given up to two loops by [23]

$\lambda_{K}=\frac{\alpha_{s}}{\pi} C_{F}+\left(\frac{\alpha_{s}}{\pi}\right)^{2} C_{F}\left[C_{A}\left(\frac{67}{36}-\frac{\pi^{2}}{12}\right)-\frac{5}{18} n_{f}\right]$, with $C_{A}=3$ being a color factor and $n_{f}$ the number of quark flavors. We allow the scale $\mu$ to evolve to $P_{1}^{+} / \bar{N}$ in $K$ and to $2 v^{2} P_{2}^{-} / \sqrt{e}$ in $G$, obtaining the RG solution of $K+G$,

$$
\begin{aligned}
& K\left(\frac{P_{1}^{+}}{\mu \bar{N}}, \alpha_{s}(\mu)\right)+G\left(\frac{2 v^{2} P_{2}^{-}}{\sqrt{e} \mu}, \alpha_{s}(\mu)\right) \\
& =K\left(1, \alpha_{s}\left(P_{1}^{+} / \bar{N}\right)\right)+G\left(1, \alpha_{s}\left(2 v^{2} P_{2}^{-} / \sqrt{e}\right)\right) \\
& \quad-\int_{P_{1}^{+} / \bar{N}}^{2 v^{2} P_{2}^{-} / \sqrt{e}} \frac{d \mu}{\mu} \lambda_{K}\left(\alpha_{s}(\mu)\right) \\
& =-\int_{P_{1}^{+} / \bar{N}}^{2 v^{2} P_{2}^{-} / \sqrt{e}} \frac{d \mu}{\mu} \lambda_{K}\left(\alpha_{S}(\mu)\right) .
\end{aligned}
$$

Substituting the above evolution kernel into Eq. (10), we solve for the jet function

$$
\begin{aligned}
\tilde{J}(N)= & \tilde{J}_{\text {in }}(N) \exp \left[-\int_{\sqrt{e} P_{1}^{+} /\left(2 \bar{N} P_{2}^{-}\right)}^{v^{2}}\right. \\
& \left.\times \frac{d \bar{v}^{2}}{2 \bar{v}^{2}} \int_{P_{1}^{+} / \bar{N}}^{2 \bar{v}^{2} P_{2}^{-} / \sqrt{e}} \frac{d \mu}{\mu} \lambda_{K}\left(\alpha_{s}(\mu)\right)\right] \\
= & \tilde{J}_{i n}(N) \exp \left[-\frac{1}{2} \int_{1 / \bar{N}}^{2 v^{2} P_{2}^{-} /\left(\sqrt{e} P_{1}^{+}\right)}\right. \\
& \left.\times \frac{d y}{y} \int_{1 / \bar{N}}^{y} \frac{d w}{w} \lambda_{K}\left(\alpha_{S}\left(w P_{1}^{+}\right)\right)\right],
\end{aligned}
$$

with the variable changes $\mu=w P_{1}^{+}$and $\bar{v}^{2}=\sqrt{e} P_{1}^{+} y /$ $\left(2 P_{2}^{-}\right)$. The initial condition $\tilde{J}_{i n}(N)$ for the jet function is determined via matching: we expand Eq. (26) to $\mathcal{O}\left(\alpha_{s}\right)$ for a fixed coupling constant, and compare it with Eq. (6) to get

$\tilde{J}_{i n}(N)=\frac{1}{N}\left[1-\frac{\alpha_{S} C_{F}}{4 \pi}\left(\frac{3 \pi^{2}}{2}-\frac{1}{4}\right)\right]$.

If an order-unity constant $C$ is introduced into the exponent, the initial condition will be modified accordingly:

$$
\begin{aligned}
\tilde{J}(N)= & \frac{1}{N}\left[1-\frac{\alpha_{S} C_{F}}{4 \pi}\left(\frac{3 \pi^{2}}{2}-\frac{1}{4}+\ln ^{2} C\right)\right] \\
& \times \exp \left[-\frac{1}{2} \int_{C / \bar{N}}^{2 v^{2} P_{2}^{-} /\left(\sqrt{e} P_{1}^{+}\right)}\right. \\
& \left.\times \frac{d y}{y} \int_{1 / \bar{N}}^{y} \frac{d w}{w} \lambda_{K}\left(\alpha_{S}\left(w P_{1}^{+}\right)\right)\right] .
\end{aligned}
$$

For a heavy-to-light transition at maximal recoil, we have $P_{1}^{+}=P_{2}^{-}=m_{B} / \sqrt{2}, m_{B}$ being the $B$ meson mass. Choosing the factor $v^{2}=1 / 2$, i.e., $\xi^{2}=Q^{2}$, and neglecting the running of the coupling constant, we derive the jet function in the Mellin space

$\tilde{J}(\bar{N})=\tilde{J}_{i n}(N) \exp \left[-\frac{\lambda_{K}}{4}\left(\ln ^{2} \bar{N}-\ln \bar{N}+\frac{1}{4}\right)\right]$.

This is the improvement of the threshold resummation with a fixed coupling constant to the NLL accuracy. The inverse 
Mellin transformation brings the jet function back to the momentum fraction space,

$$
\begin{aligned}
J(x)= & \int_{c-i \infty}^{c+i \infty} \frac{d N}{2 \pi i}(1-x)^{-N} \tilde{J}(N) \\
= & J_{0} \int_{-\infty}^{\infty} \frac{d t}{\pi}(1-x)^{\exp \left(t-\gamma_{E}+1 / 2\right)} \\
& \times \sin \left(\frac{\lambda_{K} \pi t}{2}\right) \exp \left(-\frac{\lambda_{K} t^{2}}{4}\right),
\end{aligned}
$$

with the coefficient

$$
J_{0}=-\left[1-\frac{\alpha_{S} C_{F}}{4 \pi}\left(\frac{3 \pi^{2}}{2}-\frac{1}{4}\right)\right] \exp \left(\frac{\lambda_{K} \pi^{2}}{4}\right) .
$$

In the above formula $c$ is an arbitrary real constant larger than the real parts of all the poles of the integrand, the variable change $N=\exp (t+i \pi)(N=\exp (t-i \pi))$ has been adopted for the piece of contour above (below) the branch cut in Fig. 3 of [15], and the further variable change $t+\gamma_{E}-1 / 2 \rightarrow t$ has been made. It is found that Eq. (30) exhibits the features similar to those of the LL jet function [15]: it vanishes as $x \rightarrow 0$ and $x \rightarrow 1$, and it is normalized to unity up to corrections of $\mathcal{O}\left(\alpha_{s}\right)$.

For the numerical analysis to be performed in the next section, we take into account the running effect of the coupling constant by inserting $\alpha_{s}(\mu)=4 \pi /\left[\beta_{0} \ln \left(\mu^{2} / \Lambda_{\mathrm{QCD}}^{2}\right)\right]$ into Eq. (26) with $\beta_{0}=11-2 n_{f} / 3$ and the QCD scale $\Lambda_{\mathrm{QCD}}$. Note that Eq. (26) vanishes as $\bar{N}$ approaches to the Landau pole, $\bar{N} \rightarrow P_{1}^{+} / \Lambda_{\mathrm{QCD}}$, namely, as $N \approx 8.4$ for $m_{B}=5.28$ $\mathrm{GeV}$ and $\Lambda_{\mathrm{QCD}}=0.25 \mathrm{GeV}$ [18]. This singularity has to be smeared in some way, when the inverse Mellin transformation is applied.

\section{Numerical results}

In this section we examine the NLL threshold resummation effect on various $\mathrm{CP}$ asymmetries in the $B \rightarrow K \pi$ decays. The first step is to convert the jet function in the Mellin space to the momentum fraction space, which is usually done through the inverse Mellin transformation defined by the first line of Eq. (30). Due to the existence of the Landau pole, an extrapolation of Eq. (26) in the large $N$ region is necessary for avoiding this singularity, which then introduces theoretical uncertainty. On the other hand, it has been observed [24] that the threshold resummation effect is mainly governed by the behavior of Eq. (26) at intermediate $N$ for currently accessible energy scales. Therefore, we will employ the best fit method proposed in [14], instead of the inverse Mellin transformation: the Mellin transformation of a parametrized jet function is fit to Eq. (26) in the intermediate $N$ region.

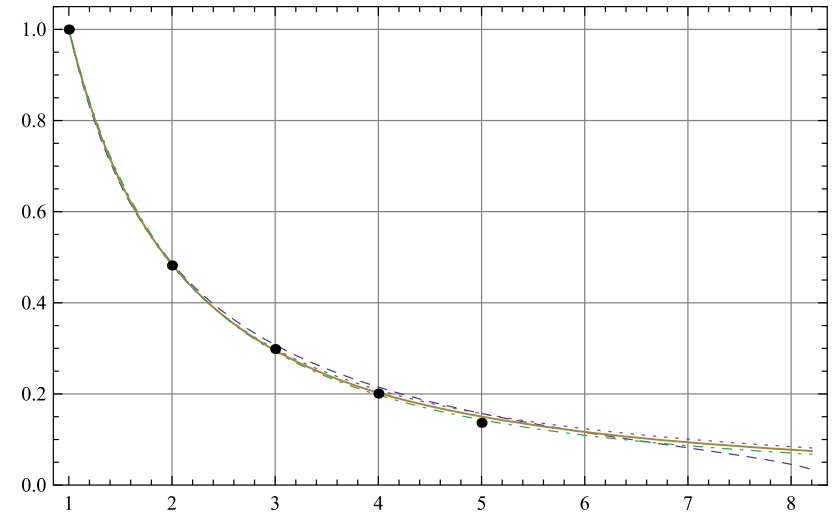

Fig. 4 Jet functions fitted to Eq. (26) with LO $\tilde{J}_{i n}$ in the ranges $N=1$ 3 (dotted line), $N=1-4$ (solid line), and $N=1-5$ (dash-dotted line), where the horizontal (vertical) axis is labelled by $N(\tilde{J}(N))$. The exact $N$ dependence of Eq. (26) (dashed line) is also displayed for comparison

We parametrize the jet function in the momentum fraction space as

$J(x)=\frac{\Gamma(\alpha+\beta+2)}{\Gamma(\alpha+1) \Gamma(\beta+1)} x^{\alpha}(1-x)^{\beta}$,

which is motivated by the feature of Eq. (30), i.e., vanishing as $x \rightarrow 0$ and $x \rightarrow 1$. The prefactor has been introduced to obey the normalization $\int d x J(x)=\tilde{J}(1)=1$. It means that we have chosen the initial condition at LO, $\tilde{J}_{i n}(N)=1 / N$, since we intend to focus on effects from the resummation. We mention that Eq. (26) is roughly, but not exactly, equal to unity as $N=1$ even with the LO $\tilde{J}_{i n}(N)$. The equality can be made exact by choosing $v^{2}=\exp \left(1 / 2-\gamma_{E}\right) / 2 \approx 0.47$, quite close to $v^{2}=1 / 2$ taken in this work. The Mellin transformation of Eq. (32) is then fit to Eq. (26) in the intermediate $N$ region, and its deviation from Eq. (26) at large $N$ is regarded as an extrapolation to avoid the Landau singularity.

The best fits to Eq. (26) for $n_{f}=4$ in the ranges from $N=1$ to $3, N=1$ to 4 , and $N=1$ to 5 produce the curves displayed in Fig. 4, which exhibit good agreement with Eq. (26) at intermediate $N$, and start to deviate from Eq. (26) as $N>6$. We take the jet function from the $N=1$ 4 fit with the parameters $\alpha=0.58$ and $\beta=0.47$ to generate our results, and those from the $N=1-3$ fit ( $\alpha=0.43$ and $\beta=0.33)$ and from the $N=1-5$ fit $(\alpha=0.76$ and $\beta=0.67)$ to estimate the theoretical uncertainty. The similarity among the three fitted jet functions suggests that the uncertainty from avoiding the Landau pole is not severe in our best fit method. Compared to the LL jet function $J(x) \propto[x(1-x)]^{0.3}[14]$, the NLL one provides stronger suppression at the end points of $x$, with which particles involved in the hard decay kernels tend to be more off-shell, and the perturbative analysis of the $B \rightarrow K \pi$ decays is expected to be more reliable.

Because the threshold logarithms mainly arise from the loop corrections to the weak vertex in an exclusive $B$ meson 
Table 1 Direct and mixing-induced CP asymmetries in the $B \rightarrow K \pi$ decays

\begin{tabular}{llllll}
\hline & Data & LL & NLL $(N=1-3)$ & NLL $(N=1-4)$ & NLL $(N=1-5)$ \\
\hline$A_{C P}\left(K^{0} \pi^{-}\right)$ & $-0.017 \pm 0.016$ & -0.009 & -0.009 & -0.011 & -0.013 \\
$A_{C P}\left(K^{-} \pi^{0}\right)$ & $0.037 \pm 0.021$ & -0.0020 & 0.0024 & -0.0028 & -0.0074 \\
$A_{C P}\left(K^{-} \pi^{+}\right)$ & $-0.083 \pm 0.004$ & -0.106 & -0.080 & -0.085 & -0.088 \\
$A_{C P}\left(K^{0} \pi^{0}\right)$ & $0.00 \pm 0.13$ & -0.079 & -0.082 & -0.083 & -0.083 \\
$S_{K^{0} \pi^{0}}$ & $0.58 \pm 0.17$ & 0.71 & 0.70 & 0.70 & 0.71 \\
\hline
\end{tabular}

decay $[15,17]$, we include the relevant next-to-leading-order (NLO) corrections to a hard kernel, i.e., the NLO Wilson coefficients and the vertex corrections [18], for consistency. The factorization formulas for the $B \rightarrow K \pi$ decay amplitudes with the NLO hard kernels are referred to [18]. The hadronic inputs, including meson masses and decay constants, meson distribution amplitudes, chiral scales $m_{0}$ associated with the pion and kaon twist-3 distribution amplitudes, and the QCD scale $\Lambda_{\mathrm{QCD}}$ are also the same as in [18]. For the Cabibbo-Kobayashi-Maskawa matrix elements, we take the Wolfenstein parametrization with the values $A=$ $0.836 \pm 0.015, \lambda=0.22453 \pm 0.00044, \bar{\rho}=0.122_{-0.017}^{+0.018}$ and $\bar{\eta}=0.355_{-0.011}^{+0.012}[25]$.

The outcomes for the $\mathrm{CP}$ asymmetries under the LL and NLL threshold resummations are listed in Table 1, in which the values in the column labelled by LL match well the corresponding ones in [18]. It is found that the NLL effect enhances the direct $\mathrm{CP}$ asymmetries $A_{C P}\left(K^{0} \pi^{-}\right)$ and $A_{C P}\left(K^{0} \pi^{0}\right)$ by about $20 \%$ and $5 \%$, respectively, and decreases $A_{C P}\left(K^{-} \pi^{+}\right)$by about $20 \%$ relative to the LL results. The $20 \%$ difference for some modes may be attributed to that the $B$ meson mass is not huge enough to suppress the strong coupling, so the NLL effect is sizable. It is not surprise that the tiny $A_{C P}\left(K^{-} \pi^{0}\right)$ is more sensitive to the NLL effect, and even flips sign, when the jet function from the $N=1-3$ fit is employed. The mixing-induced CP asymmetry $S_{K^{0} \pi^{0}}$ is very stable with respect to various inputs of the jet function. It is understandable, since this observable is supposed to be close to $\sin \left(2 \phi_{1}\right), \phi_{1}$ being the weak phase, in penguin-dominated modes like $B \rightarrow K \pi$.

The comparison of the column labelled by NLL $(N=1-$ 4) with those labelled by NLL ( $N=1-3)$ and NLL $(N=1-$ $5)$ in Table 1 reflects the theoretical uncertainty in our calculation. The distinctions among the three columns for $A_{C P}\left(K^{-} \pi^{+}\right)$and $A_{C P}\left(K^{0} \pi^{0}\right)$ with larger magnitudes are below $10 \%$, indicating that the uncertainty is under control. The other two direct CP asymmetries are smaller, and suffer stronger uncertainties as expected. Another theoretical uncertainty originates from the next-to-next-to-leading-logarithm (NNLL) effect of the resummation, which can be probed by varying the bounds of the integration variable $y$ in Eq. (28) $[26,27]$. In fact, the difference among the three columns labelled by the various $N$ intervals can be regarded as an uncertainty beyond NLL level from this viewpoint. As a test, we choose $C=2$ for the lower bound and $v^{2}=C / 2=1$ for the upper bound to respect the $N=1$ limit, together with the same initial condition $\tilde{J}_{i n}(N)=1 / N$. It is seen that even $A_{C P}\left(K^{-} \pi^{0}\right)$, which is supposed to be most sensitive to this variation, increases by only $10 \%$. The other direct CP asymmetries just change by few percent. It hints that the NNLL effect is not significant in our formalism. We also examine the theoretical uncertainty from the QCD scale $\Lambda_{\mathrm{QCD}}$, and find that it is minor. For instance, altering $\Lambda_{\mathrm{QCD}}$ within its roughly $10 \%$ uncertainty causes only $8 \%$ and $7 \%$ changes of $A_{C P}\left(K^{-} \pi^{+}\right)$and $A_{C P}\left(K^{0} \pi^{0}\right)$, respectively.

\section{Conclusion}

In this paper we have improved the LL threshold resummation for exclusive $B$ meson decays to the NLL accuracy. The recipe contains the computation of the one-loop jet function factorized out of decay amplitudes, the derivation of the evolution kernels, the matching of the resummation formula to the one-loop jet function, and the inclusion of the running effect of the coupling constant. It has been observed that the NLL threshold resummation suppresses the end-point region with $x \sim 0$ more strongly than the LL one. Because we focused on the resummaton effect, we did not take into account the NLO piece in the initial condition of the jet function. For consistency, we worked on the PQCD factorization formulas for the $B \rightarrow K \pi$ decays with the hard kernels including the NLO Wilson coefficients and the vertex corrections. It has been explained that the different LL and NLL threshold resummation effects can be compared unambiguously through the investigation of the $\mathrm{CP}$ asymmetries. We have shown that the replacement of the LL jet function by the NLL one causes up to $20 \%$ variation of the $B \rightarrow K \pi$ direct $\mathrm{CP}$ asymmetries, which is not negligible for precision analyses of $B$ meson decays. On the contrary, the mixing-induced $\mathrm{CP}$ asymmetry almost remains untouched under the above replacement. Moreover, the theoretical uncertainty from the inverse Mellin transformation of the threshold resummation is under control. 
The implementation of the NLL threshold resummation derived here in the PQCD approach to exclusive $B$ meson decays is nontrivial, and demands more efforts. As pointed out in the Introduction, the threshold resummation modifies hard decay kernels by including partial higher order contributions, so hadron distribution amplitudes should be adjusted accordingly. In principle, it is more appropriate to execute this task in a global study of many two-body hadronic $B$ meson decay modes. A global fit to available data based on the PQCD approach with the NLL threshold resummation will be attempted in near future.

Acknowledgements We thank S. Mishima for useful discussions. This work was supported in part by NSFC under Grant No. 11347030, and by MOST of R.O.C. under Grant No. MOST-107-2119-M-001-035-MY3.

Data Availability Statement This manuscript has no associated data or the data will not be deposited. [Authors' comment: This study is purely theoretical, and thus does not yield associated experimental data].

Open Access This article is licensed under a Creative Commons Attribution 4.0 International License, which permits use, sharing, adaptation, distribution and reproduction in any medium or format, as long as you give appropriate credit to the original author(s) and the source, provide a link to the Creative Commons licence, and indicate if changes were made. The images or other third party material in this article are included in the article's Creative Commons licence, unless indicated otherwise in a credit line to the material. If material is not included in the article's Creative Commons licence and your intended use is not permitted by statutory regulation or exceeds the permitted use, you will need to obtain permission directly from the copyright holder. To view a copy of this licence, visit http://creativecomm ons.org/licenses/by/4.0/.

Funded by SCOAP ${ }^{3}$.

\section{References}

1. A. Szczepaniak, E.M. Henley, S.J. Brodsky, Phys. Lett. B 243, 287 (1990)

2. R. Ahkoury, G. Sterman, Y.P. Yao, Phys. Rev. D 50, 358 (1994)

3. M. Beneke, T. Feldmann, Nucl. Phys. B 592, 3 (2000)

4. M. Beneke, G. Buchalla, M. Neubert, C.T. Sachrajda, Phys. Rev. Lett. 83, 1914 (1999)

5. M. Beneke, G. Buchalla, M. Neubert, C.T. Sachrajda, Nucl. Phys. B 591, 313 (2000)

6. M. Beneke, G. Buchalla, M. Neubert, C.T. Sachrajda, Nucl. Phys. B 606, 245 (2001)

7. A.V. Manohar, I.W. Stewart, Phys. Rev. D 76, 074002 (2007)

8. H.N. Li, H.L. Yu, Phys. Rev. Lett. 74, 4388 (1995)

9. H.N. Li, H.L. Yu, Phys. Rev. D 53, 2480 (1996)

10. Y.Y. Keum, H.N. Li, A.I. Sanda, Phys. Lett. B 504, 6 (2001)

11. Y.Y. Keum, H.N. Li, A.I. Sanda, Phys. Rev. D 63, 054008 (2001)

12. Y.Y. Keum, H.N. Li, Phys. Rev. D 63, 074006 (2001)

13. C.D. Lu, K. Ukai, M.Z. Yang, Phys. Rev. D 63, 074009 (2001)

14. T. Kurimoto, H.N. Li, A.I. Sanda, Phys. Rev. D 65, 014007 (2002)

15. H.N. Li, Phys. Rev. D 66, 094010 (2002)

16. G.P. Korchemsky, D. Pirjol, T.M. Yan, Phys. Rev. D 61, 114510 (2000)

17. H.N. Li, K. Ukai, Phys. Lett. B 555, 197 (2003)

18. H.N. Li, S. Mishima, A.I. Sanda, Phys. Rev. D 72, 114005 (2005)

19. H.N. Li, Phys. Rev. D 55, 105 (1997)

20. H.N. Li, Phys. Part. Nucl. 45, 756 (2014)

21. H.N. Li, Y.L. Shen, Y.M. Wang, JHEP 02, 008 (2013)

22. H.N. Li, Y.L. Shen, Y.M. Wang, JHEP 01, 004 (2014)

23. J. Kodaira, L. Trentadue, Phys. Lett. B 112, 66 (1982)

24. H.L. Lai, H.N. Li, Phys. Lett. B. 471, 220 (1999)

25. M. Tanabashi et al., Particle Data Group, Phys. Rev. D 98, 030001 (2018)

26. H.N. Li, Z. Li, C.P. Yuan, Phys. Rev. Lett. 107, 152001 (2011)

27. H.N. Li, Z. Li, C.P. Yuan, Phys. Rev. D 87, 074025 (2013) 